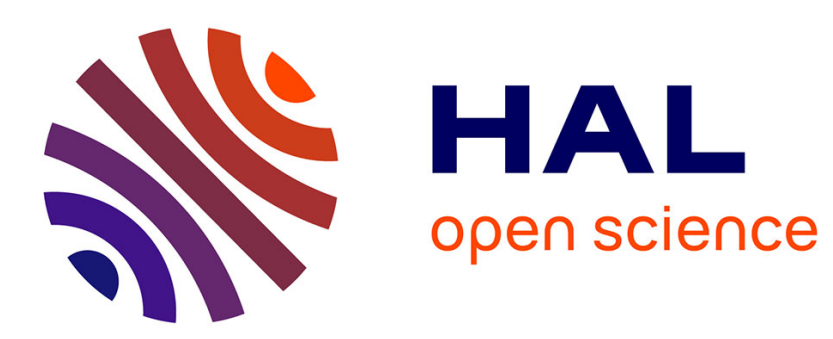

\title{
Creation of zebrafish knock-in reporter lines in the nefma gene by Cas9-mediated homologous recombination
}

Alexis Eschstruth, Sylvie Schneider-Maunoury, François Giudicelli

\section{To cite this version:}

Alexis Eschstruth, Sylvie Schneider-Maunoury, François Giudicelli. Creation of zebrafish knock-in reporter lines in the nefma gene by Cas9-mediated homologous recombination. Genesis, 2019, pp.e23340. 10.1002/dvg.23340 . hal-02315049

\section{HAL Id: hal-02315049 \\ https://hal.sorbonne-universite.fr/hal-02315049}

Submitted on 14 Oct 2019

HAL is a multi-disciplinary open access archive for the deposit and dissemination of scientific research documents, whether they are published or not. The documents may come from teaching and research institutions in France or abroad, or from public or private research centers.
L'archive ouverte pluridisciplinaire HAL, est destinée au dépôt et à la diffusion de documents scientifiques de niveau recherche, publiés ou non, émanant des établissements d'enseignement et de recherche français ou étrangers, des laboratoires publics ou privés. 


\title{
Creation of zebrafish knock-in reporter lines in the nefma gene by Cas9-mediated homologous recombination
}

\author{
Alexis Eschstruth, Sylvie Schneider-Maunoury, François Giudicelli*. \\ Sorbonne Université, CNRS UMR7622, Inserm U1156, Institut de Biologie Paris-Seine (IBPS) - \\ Laboratoire de Biologie du développement, F-75005, Paris. \\ * Present address: Institut de Biologie de l'ENS (IBENS), École normale supérieure, 75005 Paris, \\ France.
}

\begin{abstract}
CRISPR/Cas9-based strategies are widely used for genome editing in many organisms, including zebrafish. Although most applications consist in introducing double strand break (DSB)-induced mutations, it is also possible to use CRISPR/Cas9 to enhance homology directed repair (HDR) at a chosen genomic location in order to create knock-ins with optimally controlled precision.

Here, we describe the use of CRISPR/Cas9-targeted DSB followed by HDR to generate zebrafish transgenic lines where exogenous coding sequences are added in the nefma gene, in frame with the endogenous coding sequence. The resulting knock-in embryos express the added gene (fluorescent reporter or KalTA4 transactivator) specifically in the populations of neurons that express nefma, making them convenient tools for research on these populations.
\end{abstract}

\section{Keywords}

genome editing, knock-in, CRISPR/Cas9, nefma, zebrafish 


\section{Introduction}

The emergence of CRISPR/Cas9-based techniques has opened wide a range of genome editing strategies that were previously out of reach. Among these is gene replacement via homologous recombination between a Cas9-cleaved chromosome and donor DNA, a process known as homologydirected repair (HDR) (Jasin and Haber, 2016). Such strategy is of particular interest in zebrafish, since there exists no established technique for gene replacement in this model vertebrate species, in other respects particularly appropriate to genetic studies.

Previous works have demonstrated the feasibility of such approaches, using targeted TALENs or Cas9 nucleases to generate double strand breaks (DSBs) at defined chromosome loci. Most of these published methods involve short, single-stranded, oligonucleotide as donor DNA (Bedell et al., 2012; Hruscha et al., 2013; Hwang et al., 2013; Gagnon et al., 2014; Hisano et al., 2015; Armstrong et al., 2016). Although this strategy has proved efficient, it is obviously limited in that it can only replace short gene portions, typically a few nucleotides, by equally short sequences (e.g. point mutations, stop codons, loxP sites, epitope tag coding sequences, etc.).

In order to replace genomic portions on a larger scale, donor DNA has to be produced from plasmid, so as to allow longer homology arms (> $500 \mathrm{nt}$ ) and larger inserts. Few studies have reported successful HDR in zebrafish using long homology arms. The earliest cases used engineered TALEN nucleases (Hoshijima, Jurynec, and Grunwald, 2016; Shin, Chen, and Solnica-Krezel, 2014; Zu et al., 2013). More recently, similar strategy was applied using the more flexible CRISPR/Cas9 system for generating double-strand breaks (DSBs) (Irion, Krauss, and Nüsslein-Volhard, 2014; Li et al., 2015; Y. Zhang, Huang, Zhang, and Lin, 2016). However, in these latter studies, HDR was used mainly to achieve small insertions/deletions or base substitutions. Only one case described the insertion of large sequences, encoding fluorescent proteins ( $\mathrm{Li}$ et al., 2015).

The development of theses gene editing technologies (reviewed by Albadri, Del Bene, and Revenu, 2017) suggests that knock-in of large pieces of DNA by CRISPR-targeted DSB followed by HDR, although seldom described so far, likely constitutes an efficient strategy for the generation of novel useful transgenic zebrafish lines, including much needed Gal4 driver lines. So far, zebrafish Gal4 driver lines have been generated either by conventional transgenesis (reviewed in Asakawa and Kawakami, 2008), or occasionally by non-homologous end-joining (NHEJ) at CRISPR-targeted loci (Auer, Duroure, De Cian, Concordet, and Del Bene, 2014; Kimura, Hisano, Kawahara, and Higashijima, 2014), but never by HDR. Here, we describe successful knock-in of fluorescent protein and Gal4 transactivator at precise location in the nefma locus by CRISPR/Cas9-induced HDR.

\section{Results}

We chose to target our gene replacement project to the nefma gene. In situ hybridization experiments have shown that expression of nefma mRNA is both robust and precisely restricted to a subpopulation of neurons, essentially those with large axon calibre (Baraban, Anselme, Schneider-Maunoury, and Giudicelli, 2013). Due to their large size and early growth, this neuronal subpopulation is of great interest for investigation with optogenetic tools, both sensors and effectors. A prerequisite for the use of such tools is the availability of an efficient Gal4 driver line expressed in the population of interest. Although several lines expressing Gal4 in Mauthner neurons have been published (e.g. s1181t-Gal4 (Scott and Baier, 2009) and y264-Gal4 (Tabor et al., 2014)), no Gal4 driver line expressed specifically in nefma-positive, large axon calibre neurons, including both central and peripheral nervous system, has been reported yet.

Our aim was to suppress the stop codon of the nefma coding sequence so that the nefma reading frame be followed by a reporter protein coding sequence (mCherry-CaaX or KalTA4), with an intervening $2 \mathrm{~A}$ peptide separating the two components. When such bipartite mRNA is translated, ribosomes skip one peptide bond within the nascent $2 \mathrm{~A}$ peptide, resulting in two distinct protein produced in equimolar proportions from a single translation unit. Thus, the strategy aimed at leaving the nefma protein untouched, while tagging cells that express it.

We first chose a suitable CRISPR genomic target site in the vicinity of the stop codon in nefma coding sequence. Two candidate sites, respectively situated $38 \mathrm{nt}$ (gRNA1) and $83 \mathrm{nt}$ (gRNA2) 
upstream of nefma stop codon (target sequences: GAAGGTAGTCTCCAAACCAGCGG and TACCGTCACCGAGAAGGGGG $A G G$ ), were selected using Crispor (Haeussler et al., 2016), based on their position, sequence specificity and predicted cleavage efficiency. Both were tested by injecting the corresponding gRNA together with Cas9 protein in fertilised eggs, followed by systematic analysis of the target locus by heteroduplex PCR assay on the resulting embryos. Since only gRNA1 consistently showed acceptable rate of cleavage in heteroduplex PCR assay when injected in zebrafish embryos (Fig.S1), it was retained for use in the rest of the experiments.

We then designed a donor DNA construct containing the following elements (Fig.1):

- a 820-bp 5' homology arm corresponding to the part of nefma coding sequence upstream of the CRISPR site

- the remaining $54 \mathrm{nt}$ coding sequence of nefma C-terminus, with silent mutations destined to prevent both CRISPR targeting of the replacing DNA and short range recombination immediately downstream of the CRISPR site, which would lead to repair without insertion of the cassette

- the cassette to be inserted just before nefma stop codon, consisting of the sequence encoding a $2 \mathrm{~A}$ peptide followed by either

$\circ$ a sequence encoding KalTA4, a zebrafish-optimised version of the Gal4 transcriptional activator (Distel, Wullimann, and Köster, 2009)

- a sequence encoding prenylated mCherry, optimised for zebrafish codon usage

- the 3' untranslated region of nefma transcription unit (550bp) plus $1 \mathrm{~kb}$ additional genomic sequence downstream of it.

The whole construct was carried by a pBluescript backbone, flanked by symmetric instances of the same CRISPR target sequence as that used to target nefma. This was intended to allow linearisation of the plasmid and facilitate genome integration as in (Li et al., 2015).

In order to obtain HDR, we injected the donor plasmid together with gRNA and Cas9-coding, in vitro translated mRNA. Resulting embryos were then screened for fluorescence at 1,2 and 3 days post fertilisation. Patterns of fluorescence varied widely, ranging from clear neuron-specific expression in large numbers of cells to total absence of fluorescence (data not shown). Ectopic expression (typically in muscle cells or yolk enveloping layer) was rare, which may not be surprising since the constructs used were deprived of any promoter sequence, hence little liable to expression unless inserted at their intended location in the nefma locus. All injected embryos were raised to adulthood. In order to prioritise the screening, they were kept separated according to their pattern of transient expression. However, there appeared to be poor correlation between transient expression after injection and subsequent transmission of a functional allele to the progeny, presumably reflecting independent events happening in somatic and germinal lineages. By crossing $40 \mathrm{~F} 0$ fish and screening their progeny for fluorescence, we identified 2 out of 15 fish transmitting nefma:Cherry-CaaX and 2 out of 25 fish transmitting nefma:KalTA4 detected thanks to the UAS:Kaede transgene already present in the parental genome. For all four lines, the germline mosaicism, measured as the proportion of F1 embryos exhibiting fluorescence, ranged between $2 \%$ and $4 \%$. At the following generations, the engineered alleles were transmitted by F1 fish with the expected mendelian frequency.

We verified the precise sequence of the engineered alleles by performing PCR on F1 genomic DNA, with one primer specific for donor DNA and a second primer specific of the nefma locus, beyond the homology arm of the donor DNA. In all 4 lines, the PCR and sequence confirmed that the targeted locus had been efficiently repaired by HDR on its 5 ' side, leading to fusion of the nefma coding sequence with 2A-KalTA4/mCherry (PCR A in Fig.S2). A few nucleotide differences between genomic and plasmid sequences in the 5' homology arm allowed us to determine that in 3 cases out of 4, recombination had taken place between -820 and $-440 \mathrm{nt}$ before DSB; in the fourth case, recombination had taken place between -170 et $-3 \mathrm{nt}$ before DSB. We also generated double carriers by crossing KalTA4 and Cherry fish with each other and genotyped by PCR the presence of the nefma wild type allele in the resulting embryos. In all double carriers displaying both green (Kaede) and red (Cherry) fluorescence, nefma wild type allele was lost, unambiguously indicating that all lines considered are true insertions at the nefma locus (PCR B in Fig.S2). This result excludes the possibility that the positive results seen by PCR encompassing the homology arm may be due to a previously described PCR artifact (Won and Dawid, 2017) rather than knock-in insertions. 
Although HDR could theoretically occur also on the 3' side of the DSB, using homology between nefma 3'UTR sequences present both in the construct and at the targeted locus, this happened in none of the four lines. Instead, repair at the 3' side was apparently accomplished by NHEJ, with insertion of various portions of plasmid DNA downstream of the gene. This means that recombination on one side does not necessarily coincide with recombination on the other side. Therefore, applications that would require recombination on both sides may need an appropriate selection process to be applied.

Live embryos carrying the engineered knocked-in alleles were easily identifiable due to red (mCherry, Fig.2A,B) or green (Kaede, Fig.2C-F) fluorescence in neurons of the CNS and cranial ganglia, starting between 2 and 2,5 dpf. The pattern of fluorescent neurons globally matched known expression of nefma, with one notable exception: both nefma:KalTA4 engineered lines showed strong expression of UAS reporters in scattered cells of the so-called 'polster' (arrowheads in Fig2C-D), primordium of the future hatching gland (Inohaya et al., 1997). This expression, visible as soon as $1 \mathrm{dpf}$ (Fig.2C) persisted within the hatching gland structure until 3 dpf (arrowhead in Fig.2D), after which it was no longer visible, consistent with the disappearance of the gland itself. It does not correspond to a site of expression of nefma, which is strictly neuronal. Furthermore, none of the nefma:Cherry lines showed any expression in these cells. This unexpected ectopic expression might therefore result from a cryptic enhancer or promoter carried within the KalTA4 transgene sequence or from another unknown cause.

Apart from this unexpected ectopic expression, all 4 trangenes exhibited the same cellular pattern, with expression strictly restricted to the nervous system, including reticulospinal neurons like the Mauthner pair, diencephalic descending neurons, cranial ganglionic neurons of the trigeminal ganglion and lateral line ganglia, spinal cord interneurons and motor neurons (Fig.2 and 3). It should be noted however that neither KalTA4 line, through their UAS:Kade effector, seemed to reflect the tremendous expression level which noticeably characterizes Mauthner neurons when visualising nefma or nefma:Cherry expression (compare Fig.2B with Fig.2F). This might be a side effect of the two-step nature of the Gal4-UAS transactivation system.

In order to confirm that the knocked-in protein display the same expression pattern as nefma, we performed immunolabelling against mCherry in nefma:cherry-CAAX, together with the neurofilament marker 3A10, which decorates the processes of nefma-expressing neurons (Hatta, Bremiller, Westerfield, and Kimmel, 1991). The mCherry-positive cells appeared to be essentially the same as the 3A10-positive cells (Fig. 3), although their subcellular localisation differed, as expected, since 3A10 labels cytoplasmic neurofilaments while mCherry-CAAX is predominantly submembranous due to farnesylation of the $\mathrm{C}$-terminal cytosine residue.

The constructs have been intended not to alter nefma normal function, since the native nefma protein should still be produced from the modified allele. However, whether the modified nefma alleles do provide full wild type function was not directly assayed

In conclusion, we confirm that HDR mechanisms can be harnessed as a method to introduce precise modifications, including large size insertions, in the zebrafish genome. This extends the range of opportunities already offered by CRISPR/Cas9-based technologies for genome editing in zebrafish and possibly in other species.

\section{MATERIAL AND METHODS}

\section{Zebrafish lines and husbandry}

Zebrafish were raised and maintained as described (C. B. Kimmel, Ballard, Kimmel, Ullmann, and Schilling, 1995). All experiments were conducted in agreement with the European Directive 210/63/EU on the protection of animals used for scientific purposes, and the French application decree 'Décret 2013-118'. The fish facility has been approved by the French 'Service for animal protection and health', with approval number A-75-05-25. Wild-type and UAS:Kaede transgenic (rk8Tg; Hatta, Tsujii, and Omura, 2006) strains were used. The nefma:KalTA4 and nefma:mCherry_CaaX knock-in lines will be available to the academic research community upon acceptance of the manuscript. 


\section{Plasmid design and construction}

A base version of the donor construct was synthesised by GeneCust, with left and right homology arms, P2A sequence, and mCherry_CaaX coding sequence. The mCherry_CaaX sequence optimised for zebrafish codon usage was designed using the online codon optimization tool provided by Integrated DNA Technologies (http://eu.idtdna.com/CodonOpt).

Then, the KalTA4 version of the construct was generated by PstI/XmaI substitution of P2AmCherry_CaaX with E2A-KalTA4 from plasmid eGFPbait-E2A-KalTA4-pA (Addgene \#61069, Auer et al., 2014).

\section{gRNA and Cas9 mRNA preparation}

The CRISPOR online tool (http://crispor.tefor.net; Haeussler et al., 2016) was used to select potential CRISPR targets near the end of the nefma gene coding sequence, based on high specificity and predicted efficiency. 2 candidate targets sites were tested on zebrafish embryos, and cutting efficiency assessed for each of them by T7E1 test (Mashal, Koontz, and Sklar, 1995).

gRNA transcription templates were obtained by PCR using T7_Nefma_G1_Fw primer (5'GAAATTAATACGACTCACTATAGGAAGGTAGTCTCCAAACCAGGTTTTAGAGCTAGAAAT AGC-3') or T7_Nefma_G2_Fw primer (5'-

GAAATTAATACGA $\bar{C}$ TCACTATAGGTACCGTCACCGAGAAGGGGGGTTTTAGAGCTAGAA ATAGC

-3') as forward primer and sgRNA_R universal primer (5'-

AAAAGCACCGACTCGGTGCCACTTTTTCAAGTTGATAACGGACTAGCCTTATTTTAACTTG

CTATTTCTAGCTCTAAAAC-3') as reverse primer. sgRNAs were transcribed using Megascript T7

Transcription Kit (Thermo Fisher Scientific, Waltham, MA, USA ) and purified using NucleoSpin ${ }^{\circledR}$

RNA Clean up XS kit (Machery Nagel, Düren, Germany)

Cas9 mRNA was obtained by transcription from pT3TSnCas9n (Addgene 46757) (Jao, Wente, and

Chen, 2013) using mMessage mMachine T3 Transcription Kit (Thermo Fisher Scientific).

\section{Microinjection}

Zebrafish embryos were all injected at one cell stage. For gRNA efficiency assessment, sgRNA:Cas9 RNP complex was obtain by incubating Cas 9 protein (gift of JP Concordet) $(7.5 \mu \mathrm{M})$ with sgRNA $(10 \mu \mathrm{M})$ in $20 \mathrm{mM}$ Hepes- $\mathrm{NaOH} \mathrm{pH} \mathrm{7.5,150} \mathrm{mM} \mathrm{KCl}$ for $10 \mathrm{~min}$ at $28^{\circ} \mathrm{C} .1$ to $2 \mathrm{~nL}$ was injected per embryo. For knock-in experiments, embryos where injected with 150-200 pg gRNA, 250 pg Cas9 mRNA and 30 pg donor plasmid DNA prepared with Qiagen plasmid midiprep kit.

The use of Cas9 protein for tests stems from the need of getting maximal efficiency as early as possible, while that of in vitro transcribed mRNA for knock-in aims at getting later efficiency of Cas9, in order to multiply the events of DSB and subsequent repair so as to maximise the chances of obtaining HDR.

\section{Zebrafish embryo genomic DNA extraction}

Single embryos were digested for $1 \mathrm{~h}$ at $55^{\circ} \mathrm{C}$ in $40 \mu \mathrm{L}$ lysis buffer $(100 \mathrm{mM}$ Tris-Hcl $\mathrm{pH} 7.5,1 \mathrm{mM}$ EDTA, $250 \mathrm{mM} \mathrm{NaCl}, 0.2 \% \mathrm{SDS}, 0.1 \mu \mathrm{g} / \mu \mathrm{L}$ Proteinase K). Proteinase $\mathrm{K}$ was then inactivated at $95^{\circ} \mathrm{C}$ for $10 \mathrm{~min}$. Samples were centrifuged and supernatant was diluted 5 times in distilled water. $1 \mu \mathrm{L}$ was used in $25 \mu \mathrm{L}$ PCR reactions.

\section{Screening and genotyping}

Injected (F0) fish were screened for germline transmission by crossing with wild type fish and detecting fluorescence in their progeny. Two independent lines, originating from two independent F0 fish, were identified for each construct. 
For genotyping and sequencing the recombined alleles (PCR A in Fig.S2), PCR amplification was performed on DNA extracted from the tail fin of transgenic fish, with primers

ACCCAAGCCAAAGTAGCCTCTG (forward : in genomic nefma coding sequence, upstream of the homology arm used in the donor DNA constructs) and CTCAGTAGCTTTGACGGCTTGCT (reverse : in the common sequence immediately following of the CRISPR target site in both donor DNA constructs, but not present in the wild type genomic sequence).

For confirmation of insertion at the nefma locus, the 4 lines were crossed with each other, pairwise : one KalTA4 line with one Cherry line, the other KalTA4 line with the other Cherry line. For both crosses, the resulting progeny was screened at 3-4 $\mathrm{dpf}$ for fluorescence. Red fluorescence indicated the presence of the nefma:Cherry line, green fluorescence indicated the presence of the nefma:KalTA4 line together with the UAS:Kaede reporter transgene. The embryos were separated according to their fluorescence status and individually genotyped by PCR. The primers used to detect wild-type and transgenic nefma alleles (PCR B in Fig.S2) were CGAAAGCCCAACCAAAGACG (forward) and GACCATGAGCTATTTTCACCATCA (reverse), yielding expected products of 808 bp for wild-type nefma, $1591 \mathrm{bp}$ for nefma:KalTA4, $1774 \mathrm{bp}$ for nefma:Cherry.

\section{Immunostaining}

After overnight fixation in cold 4\% PFA, transgenic embryos were dehydrated in methanol, then progressively rehydrated, depigmented in a $0.1 \mathrm{M} \mathrm{KOH} / 0.6 \% \mathrm{H} 2 \mathrm{O} 2$ solution, then processed for immunochemistry using the following antibodies: rabbit anti-dsRed (Clontech), 3A10 mouse monoclonal (Developmental Studies Hybridoma Bank), Alexa488-coupled anti-mouse IgG1 (Molecular Probes), Alexa594-coupled anti-rabbit IgG (Molecular Probes). Image acquisition was done on a Leica SP5 scanning confocal microscope.

\section{ACKNOWLEDGEMENTS}

We are grateful to the zebrafish facility of the IBPS (Institut de Biologie Paris-Seine FR3631, Sorbonne Université, CNRS, Paris, France) for their technical assistance. We thank Jean-Paul Concordet (MNHN, Paris, France) for the kind gift of the Cas9 protein and Filippo del Bene (Institut Curie, Paris, France) for providing the KalTA4 plasmid. This work was supported by funding from the Centre National de la Recherche Scientifique (CNRS) and Sorbonne Université. The authors declare no competing financial interests. 


\section{REFERENCES}

Albadri, S., Del Bene, F., and Revenu, C. (2017). Genome editing using CRISPR/Cas9-based knockin approaches in zebrafish. Methods (San Diego, Calif), 121-122, 77-85.

http://doi.org/10.1016/j.ymeth.2017.03.005

Armstrong, G. A. B., Liao, M., You, Z., Lissouba, A., Chen, B. E., and Drapeau, P. (2016). Homology Directed Knockin of Point Mutations in the Zebrafish tardbp and fus Genes in ALS Using the CRISPR/Cas9 System. PloS One, 11(3), e0150188. http://doi.org/10.1371/journal.pone.0150188

Asakawa, K., and Kawakami, K. (2008). Targeted gene expression by the Gal4-UAS system in zebrafish. Development, Growth \& Differentiation, 50(6), 391-399. http://doi.org/10.1111/j.1440169X.2008.01044.X

Auer, T. O., Duroure, K., De Cian, A., Concordet, J.-P., and Del Bene, F. (2014). Highly efficient CRISPR/Cas9-mediated knock-in in zebrafish by homology-independent DNA repair. Genome Research, 24(1), 142-153. http://doi.org/10.1101/gr.161638.113

Baraban, M., Anselme, I., Schneider-Maunoury, S., and Giudicelli, F. (2013). Zebrafish Embryonic Neurons Transport Messenger RNA to Axons and Growth Cones In Vivo. The Journal of Neuroscience : the Official Journal of the Society for Neuroscience, 33(40), 15726-15734. http://doi.org/10.1523/JNEUROSCI.1510-13.2013

Bedell, V. M., Wang, Y., Campbell, J. M., Poshusta, T. L., Starker, C. G., Krug, R. G., et al. (2012). In vivo genome editing using a high-efficiency TALEN system. Nature, 491(7422), 114-118. http://doi.org/10.1038/nature11537

Distel, M., Wullimann, M. F., and Köster, R. W. (2009). Optimized Gal4 genetics for permanent gene expression mapping in zebrafish. Proceedings of the National Academy of Sciences of the United States of America, 106(32), 13365-13370. http://doi.org/10.1073/pnas.0903060106

Gagnon, J. A., Valen, E., Thyme, S. B., Huang, P., Ahkmetova, L., Pauli, A., et al. (2014). Efficient mutagenesis by Cas9 protein-mediated oligonucleotide insertion and large-scale assessment of single-guide RNAs. PloS One, 9(5), e98186. http://doi.org/10.1371/journal.pone.0098186

Haeussler, M., Schönig, K., Eckert, H., Eschstruth, A., Mianné, J., Renaud, J.-B., et al. (2016). Evaluation of off-target and on-target scoring algorithms and integration into the guide RNA selection tool CRISPOR. Genome Biology, 17(1), 148. http://doi.org/10.1186/s13059-016-1012-2

Hatta, K., Bremiller, R., Westerfield, M., and Kimmel, C. B. (1991). Diversity of expression of engrailed-like antigens in zebrafish. Development (Cambridge, England), 112(3), 821-832.

Hatta, K., Tsujii, H., and Omura, T. (2006). Cell tracking using a photoconvertible fluorescent protein. Nature Protocols, 1(2), 960-967. http://doi.org/10.1038/nprot.2006.96

Hisano, Y., Sakuma, T., Nakade, S., Ohga, R., Ota, S., Okamoto, H., et al. (2015). Precise in-frame integration of exogenous DNA mediated by CRISPR/Cas9 system in zebrafish. Scientific Reports, 5, 8841. http://doi.org/10.1038/srep08841

Hoshijima, K., Jurynec, M. J., and Grunwald, D. J. (2016). Precise Editing of the Zebrafish Genome Made Simple and Efficient. Developmental Cell, 36(6), 654-667. http://doi.org/10.1016/j.devcel.2016.02.015

Hruscha, A., Krawitz, P., Rechenberg, A., Heinrich, V., Hecht, J., Haass, C., and Schmid, B. (2013). Efficient CRISPR/Cas9 genome editing with low off-target effects in zebrafish. Development (Cambridge, England), 140(24), 4982-4987. http://doi.org/10.1242/dev.099085

Hwang, W. Y., Fu, Y., Reyon, D., Maeder, M. L., Kaini, P., Sander, J. D., et al. (2013). Heritable and precise zebrafish genome editing using a CRISPR-Cas system. PloS One, 8(7), e68708.

http://doi.org/10.1371/journal.pone.0068708 
Inohaya, K., Yasumasu, S., Araki, K., Naruse, K., Yamazaki, K., Yasumasu, I., et al. (1997). Speciesdependent migration of fish hatching gland cells that express astacin-like proteases in common [corrected]. Development, Growth \& Differentiation, 39(2), 191-197.

Irion, U., Krauss, J., and Nüsslein-Volhard, C. (2014). Precise and efficient genome editing in zebrafish using the CRISPR/Cas9 system. Development (Cambridge, England), 141(24), 48274830. http://doi.org/10.1242/dev.115584

Jao, L.-E., Wente, S. R., and Chen, W. (2013). Efficient multiplex biallelic zebrafish genome editing using a CRISPR nuclease system. Proceedings of the National Academy of Sciences of the United States of America, 110(34), 13904-13909. http://doi.org/10.1073/pnas.1308335110

Jasin, M., and Haber, J. E. (2016). The democratization of gene editing: Insights from site-specific cleavage and double-strand break repair. DNA Repair, 44, 6-16. http://doi.org/10.1016/j.dnarep.2016.05.001

Kimmel, C. B., Ballard, W. W., Kimmel, S. R., Ullmann, B., and Schilling, T. F. (1995). Stages of embryonic development of the zebrafish. Developmental Dynamics : an Official Publication of the American Association of Anatomists, 203(3), 253-310. http://doi.org/10.1002/aja.1002030302

Kimura, Y., Hisano, Y., Kawahara, A., and Higashijima, S.-I. (2014). Efficient generation of knock-in transgenic zebrafish carrying reporter/driver genes by CRISPR/Cas9-mediated genome engineering. Scientific Reports, 4, 6545. http://doi.org/10.1038/srep06545

Li, J., Zhang, B.-B., Ren, Y.-G., Gu, S.-Y., Xiang, Y.-H., and Du, J.-L. (2015). Intron targetingmediated and endogenous gene integrity-maintaining knockin in zebrafish using the CRISPR/Cas9 system. Cell Research, 25(5), 634-637. http://doi.org/10.1038/cr.2015.43

Mashal, R. D., Koontz, J., and Sklar, J. (1995). Detection of mutations by cleavage of DNA heteroduplexes with bacteriophage resolvases. Nature Genetics, 9(2), 177-183. http://doi.org/10.1038/ng0295-177

Scott, E. K., and Baier, H. (2009). The cellular architecture of the larval zebrafish tectum, as revealed by gal4 enhancer trap lines. Frontiers in Neural Circuits, 3, 13. http://doi.org/10.3389/neuro.04.013.2009

Shin, J., Chen, J., and Solnica-Krezel, L. (2014). Efficient homologous recombination-mediated genome engineering in zebrafish using TALE nucleases. Development (Cambridge, England), 141(19), 3807-3818. http://doi.org/10.1242/dev.108019

Tabor, K. M., Bergeron, S. A., Horstick, E. J., Jordan, D. C., Aho, V., Porkka-Heiskanen, T., et al. (2014). Direct activation of the Mauthner cell by electric field pulses drives ultrarapid escape responses. Journal of Neurophysiology, 112(4), 834-844. http://doi.org/10.1152/jn.00228.2014

Won, M., and Dawid, I. B. (2017). PCR artifact in testing for homologous recombination in genomic editing in zebrafish. PloS One, 12(3), e0172802. http://doi.org/10.1371/journal.pone.0172802

Zhang, Y., Huang, H., Zhang, B., and Lin, S. (2016). TALEN- and CRISPR-enhanced DNA homologous recombination for gene editing in zebrafish. Methods in Cell Biology, 135, 107-120. http://doi.org/10.1016/bs.mcb.2016.03.005

Zu, Y., Tong, X., Wang, Z., Liu, D., Pan, R., Li, Z., et al. (2013). TALEN-mediated precise genome modification by homologous recombination in zebrafish. Nature Methods, 10(4), 329-331. http://doi.org/10.1038/nmeth.2374 


\section{FIGURE LEGENDS}

Figure 1 : Scheme of the knock-in strategy

(A) a) structure of the targeted nefma genomic locus and position of the chosen CRISPR target site (orange arrowhead)

b) nefma gene knock-in construct contain essentially 3 elements assembled as follows:

i) $820 \mathrm{bp}$ of nefma genomic sequence upstream of the chosen CRISPR target site (5', homology arm)

ii) the desired cds fusion downstream of nefma cds

iii) nefma 3'UTR sequence followed by $1 \mathrm{~kb}$ of downstream genomic sequence

At the junction between $i$ and ii, corresponding to the genomic CRISPR target site, translationally silent mutations are introduced so as to prevent CRISPR targeting to the recombined allele.

(B) Genomic sequence of the C-terminal region of nefma cds, aligned with sequences of the knock-in constructs, which also represent the sequence of the recombined alleles after successful knock-in. The CRISPR target sequence is highlighted in orange, with the CGG PAM sequence underlined. Position of the predicted Cas9-induced DSB is indicated with an orange arrowhead. Asterisks indicate silent mutations introduced in nefma cds in the knock-in constructs. The CTCAGA recognition site for the PstI restriction enzyme used for engineering the constructs is underlined.

\section{Figure 2 : Expression of the knocked-in alleles in live embryos}

Fluorescence intensity is represented on an inverted grey scale (white $=$ low, black $=$ high). (A, B) Lateral (A) and dorsal (B) views of live 2,5 dpf transgenic Tg(nefma-2A-mCherry-CaaX) embryos. Red fluorescence indicates expression of the recombined allele in nefma-expressing neurons in the central and periphery nervous system.

(C-F) Lateral (D, E) and dorsal (C, F) views of live transgenic Tg(nefma-2A-KalTA4) ;

$\operatorname{Tg}(\mathrm{UAS}:$ Kaede) embryos. Kaede fluorescence results from expression of the knocked-in KalTA4 allele, the product of which activates the UAS reporter transgene.

(C) The nefma:KalTA4 allele is unexpectedly expressed in cells of the embryonic polster (black arrowhead) at $1 \mathrm{dpf}$

(D-F) At 2,5 dpf, apart from cells in the hatching gland cells (black arrowhead), expression of the Kaede reporter is specific to nefma-expressing neurons in the central and peripheral nervous system.

\section{Figure 3 : Expression of the nefma:Cherry transgene coincides with $3 A 10$ immunostaining}

(A-C) z-projections of scanning confocal images of a flat-mounted $3 \mathrm{dpf}$ transgenic $\operatorname{Tg}$ (nefma-2AmCherry-CaaX) embryo. Panels show immunostaining signals corresponding to anti-Cherry antibody (red in (A), color-coded intensity in (C)) and 3A10 antibody (green in (A), color-coded intensity in (B)) 


\section{SUPPLEMENTARY FIGURE LEGENDS}

\section{Supplementary Figure S1 : Test of guide RNA efficiency}

Two selected guide RNAs were tested by injection in zebrafish eggs together with Cas9 protein, followed by genomic DNA extraction at $1 \mathrm{dpf}$. For each sample, the target site was amplified by PCR and CRISPR-induced heterozygosity was assayed by T7 Endonuclease I digestion of double-strand DNA formed by successive denaturation and reannealing of the PCR products. For heteroduplexes where the 2 strands differ from each other at the position of the CRISPR target site, T7 Endonuclease I is expected to digest the $808 \mathrm{bp}$ DNA into 2 smaller fragments of 641 and $167 \mathrm{bp}$ for gRNA1, 686 and $122 \mathrm{bp}$ for gRNA2. The gel electrophoresis shows the test result for 8 randomly chosen embryos injected with gRNA1 (top row), gRNA2 (middle row) or not injected (bottom row). gRNA1 displays moderate but consistent efficiency across samples, while gRNA2 appears to have very low efficiency, which led to use gRNA1 in all subsequent experiments.

\section{Supplementary Figure S2 : Confirmation of insertion at the nefma locus}

Principle of the confirmation strategy : KalTA4 and Cherry lines were crossed with each other, generating progeny carrying either or both transgenes. Resulting embryos were screened for fluorescence at 3-4 dpf and their DNA was extracted. 2 PCR amplifications were performed on each embryo.

PCR A uses one primer in the inserted transgene and one primer in the target locus, outside of the homology arm present in the DNA donor vector. Therefore, it generates a 1056 bp product if and only if the target locus has been repaired by recombination with the donor DNA.

PCR B uses two primers on either side of the CRISPR target site. It generates a 808 bp product on the wild type nefma allele, a 1794 bp product on the Cherry allele, and a 1591 bp product on the KalTA4 allele.

Crossing nefma :KalTA4 with nefma :Cherry fish results in 4 distinct phenotypes in terms of fluorescence, corresponding to 8 possible genotypes. Their expected mendelian proportions are :

- $\quad 1 / 8$ green and red : (Cherry+, KalTA4+, UAS :Kaede+)

- $\quad 3 / 8$ red but not green : (Cherry+, KalTA4-, UAS :Kaede+), (Cherry+, KalTA4+, UAS :Kaede) or (Cherry+, KalTA4-, UAS :Kaede-)

- $\quad$ 1/8 green but not red : (Cherry-, KalTA4+, UAS :Kaede+)

- 3/8 with no fluorescence (Cherry-, KalTA4-, UAS :Kaede+), (Cherry-, KalTA4+, UAS :Kaede-) or (Cherry-, KalTA4-, UAS :Kaede-)

The 8 panels show the PCR A and B results for 32 embryos ( 8 of each phenotype) of a single clutch. 10 embryos carrying both Cherry and KalTA4 alleles ( 8 'green and red', plus 2 'red but not green') are marked with an asterisk : they all lack the nefma wild type allele, which proves that both KalTA4 and Cherry are allelic to nefma. Together with the result of PCR A, this indicates that both KalTA4 and Cherry are inserted as intended in the nefma locus.

The reciprocal cross (the other KalTA4 line with the other Cherry line) gave similar results : all embryos inheriting both the KalTA4 and the Cherry allele also lost the wild type nefma allele. 


\section{Figure 1. Strategy for CrispR-mediated knock-in at the nefma locus}

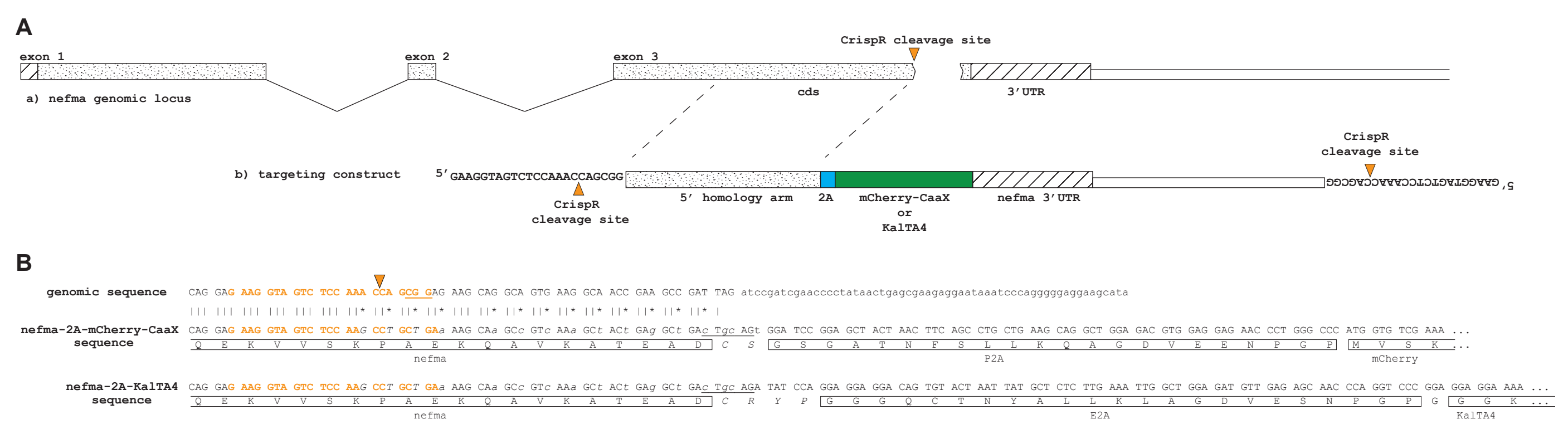


Figure 2. Expression of knocked-in nefma::mCherry-CaaX and nefma::KalTA4

A

B

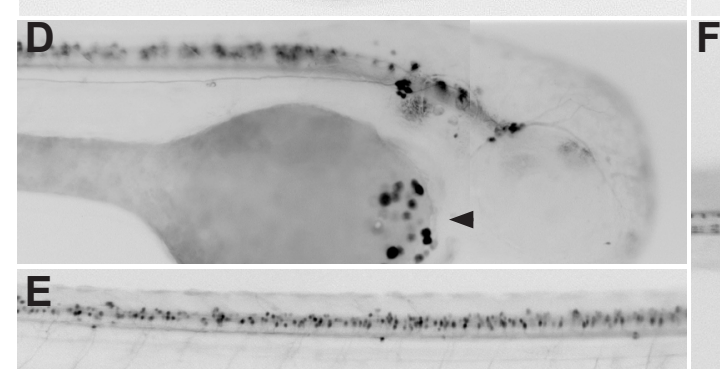

F

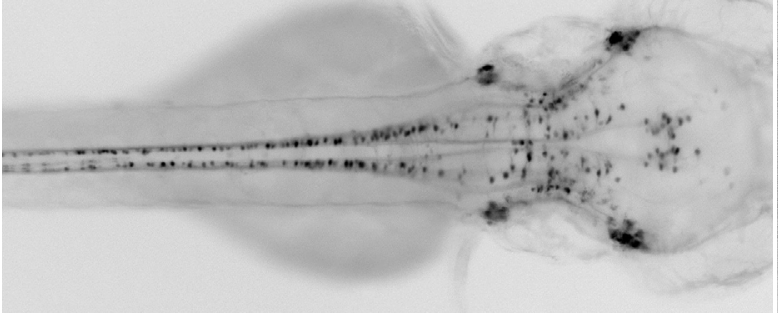

C

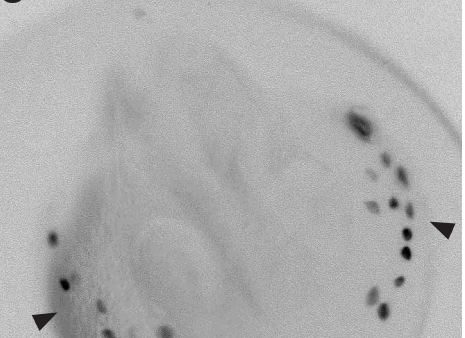


Figure 3. nefma::mCherry-CaaX expression coincides with 3A10 immunostaining
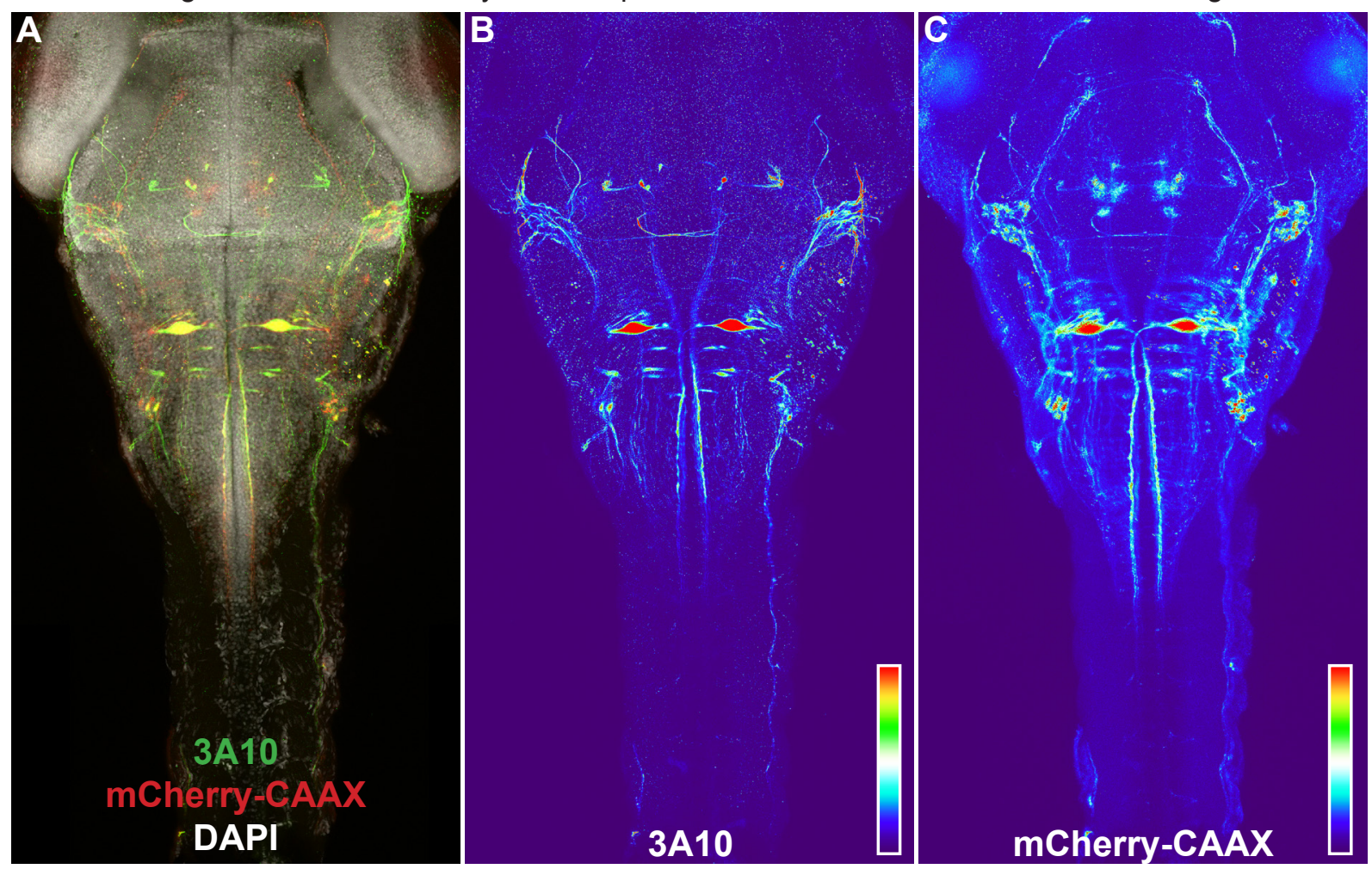
Supplementary Figure S1 : Test of guide RNA efficiency

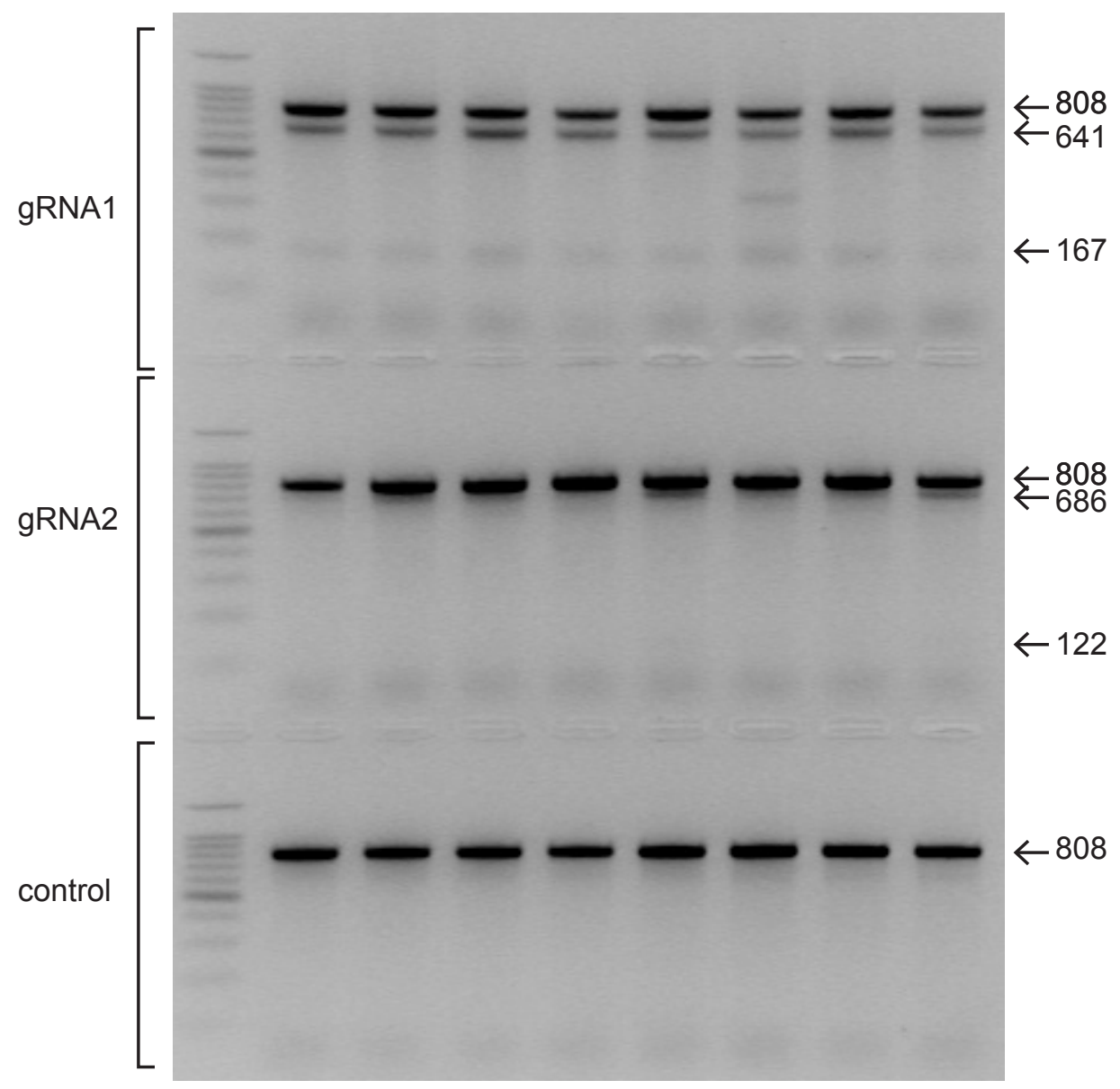




\section{Supplementary Figure S2 : Confirmation of insertion at the nefma locus}

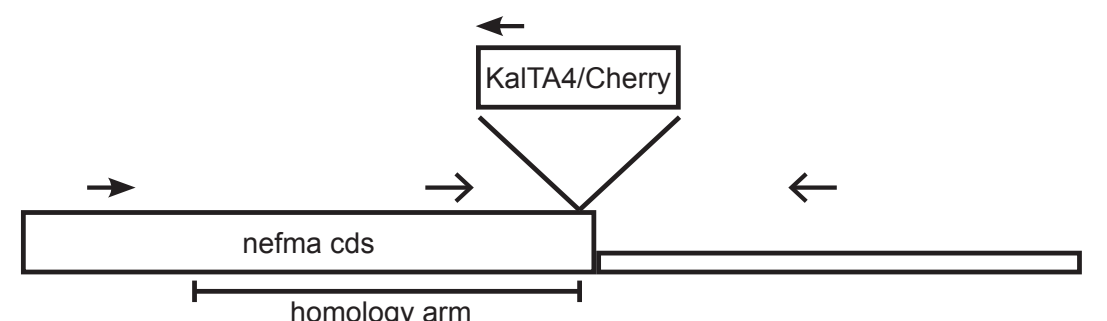

$$
\begin{aligned}
& \mathrm{PCR} A \rightarrow \ldots \ldots \leftarrow \text { wt: no product } \\
& \leftarrow \text { recombined allele : } 1056 \text { bp } \\
& \text { PCR B } \rightarrow \quad \text { wt: } 808 \text { bp } \\
& \mathrm{PCR} B \rightarrow \cdots \cdots \in \text { KalTA4: } 1591 \mathrm{bp} \\
& \text { Cherry : } 1794 \mathrm{bp}
\end{aligned}
$$

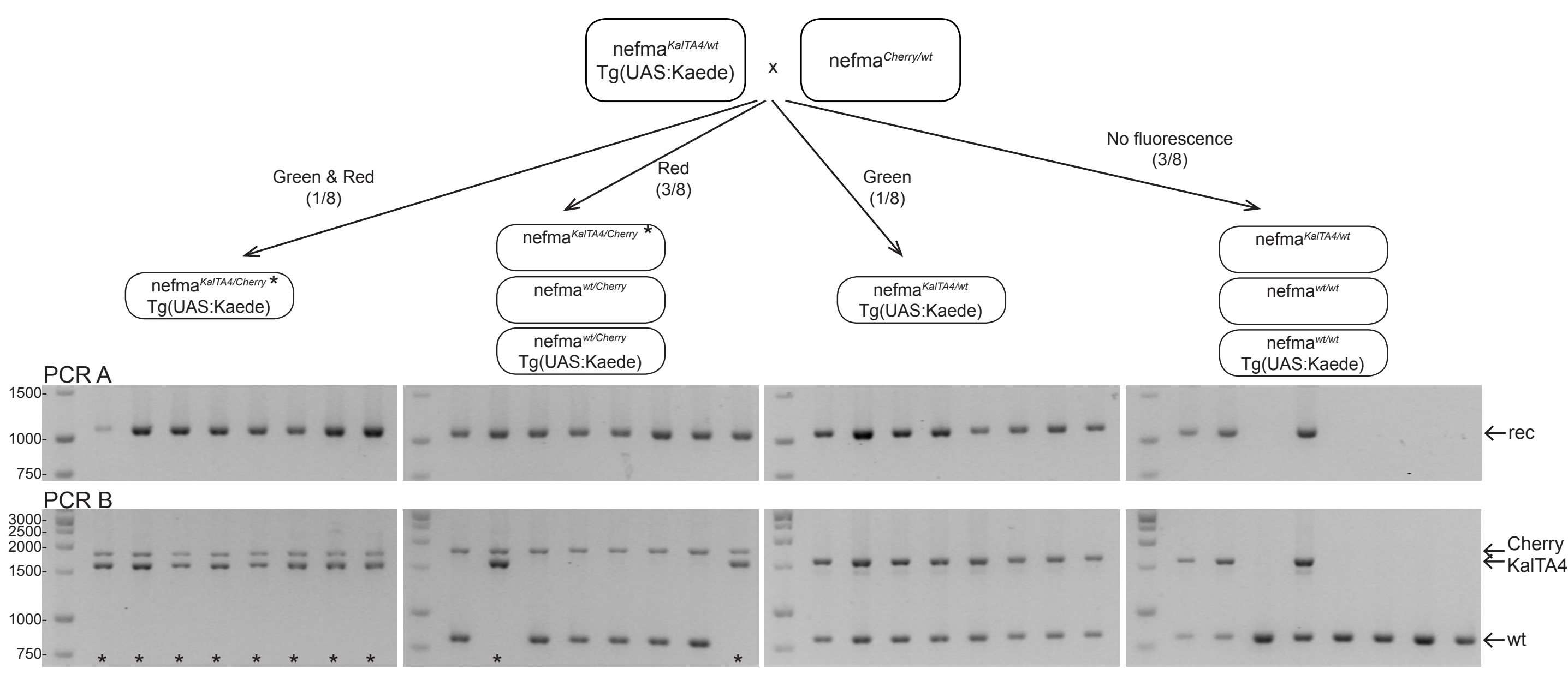

